\title{
Auditoría transfusional en un Hospital de Alta Especialidad. Análisis retrospectivo de 12 meses
}

\author{
Miguel Ángel Cano-Palmeros ${ }^{1 *}$, Jorge Castañón-González², Miguel Ángel Cano-Quevedo, \\ Dora Stephanie Morales-Uchino ${ }^{3}$, Araceli Benavides-González', Alejandro Espinosa-Kurit \\ y Diana Elvia Ruiz-Moreno ${ }^{4}$ \\ ${ }^{1}$ Servicio de Transfusión, Hospital de Alta Especialidad, Veracruz; ${ }^{2}$ Unidad de Cuidados Intensivos, Unidad de Cuidados Intensivos Hospital Juárez, \\ Ciudad de México; ${ }^{3} \mathrm{Hospital}$ de Alta Especialidad de Veracruz; ${ }^{4}$ Facultad de Medicina, Universidad Cristóbal Colón Veracruz, México
}

\begin{abstract}
Resumen
La sangre humana es la única fuente de eritrocitos, plaquetas y plasma, e incluye los factores de la coagulación. La transfusión de concentrado eritrocitario y hemoderivados es una forma simple de trasplante de órgano. Los beneficios de la transfusión sanguínea son reales, y de su uso puede depender la vida de los pacientes. Objetivo: Conocer el apego de la transfusión a las recomendaciones vigentes en el Hospital de Alta Especialidad de Veracruz. Métodos: Durante un periodo de 12 meses se llevó a cabo en el Servicio de Transfusión del Hospital de Alta Especialidad de Veracruz una auditoría sobre 3168 solicitudes de transfusión, de las cuales 2314 correspondieron a concentrado eritrocitario, 220 a concentrado plaquetario, 493 a plasma fresco congelado y 41 a crioprecipitados. Resultados y conclusiones: Se hizo un análisis de concordancia con las diferentes normas establecidas para una indicación correcta y se encontró que 2171 (67.26\%) fueron adecuadas y 1037 (32.73\%) fueron inadecuadas, lo cual traduce que la falta de formación académica en materia de medicina transfusional incide en el riesgo para los pacientes y el costo para las instituciones de salud.
\end{abstract}

PALABRAS CLAVE: Transfusión. Auditoría. Concentrado eritrocitario. Concentrado plaquetario.

\begin{abstract}
Human blood is the only source of red blood cells, platelets and plasma, and includes the clotting factors. Transfusion of concentrated erythrocyte and blood products is a simple form of organ transplant, the benefits of blood transfusion are real, and the life of the patients depends on how is used. Objective: to know the transfusion adherence to the recommendations in the Hospital of High Specialty of Veracruz, Methods: For a period of 12 months an audit took place in the Transfusion Service of the Hospital of High Specialty of Veracruz, México, on a basis of 3168 requests for transfusion from which 2314 corresponded to erythrocyte concentrate, 220 to platelet concentrate, 493 to fresh frozen plasma and 41 to cryoprecipitate. Results and conclusions: An analysis of concordance was made with the different established regulations for a right indication and the results showed that 2171 (67.26\%) were appropriate and 1037 were inadequate, which means that the lack of academic training in medicine transfusional affects the risk for patients and cost for Health Institutions.
\end{abstract}

KEY WORDS: Transfusion. Audit took. Erythrocyte concentrate. Platelet concentrate.

\author{
Correspondencia: \\ Miguel Angel Cano-Palmeros \\ Servicio de Transfusión \\ Hospital de Alta Especialidad de Veracruz \\ Av. 20 de noviembre 1074 \\ Col. Centro \\ C.P.91900, Veracruz, México \\ E-mail: mangelcanop@gmail.com
}

Fecha de recepción en versión modificada: 09-07-2016

Fecha de aceptación: 11-07-2016

DOI://dx.doi.org/10.24875/GMM.17002624
Gac Med Mex. 2017;153:765-768

Contents available at PubMed www.gacetamedicademexico.com 


\section{Introducción}

La sangre humana es la única fuente de eritrocitos, plaquetas y plasma, e incluye los factores de la coagulación. La transfusión es una forma simple de trasplante de órgano, ya que se transfiere de un donante a un paciente para corregir temporalmente una deficiencia o una alteración de una función 1 .

Los beneficios de la transfusión sanguínea son reales, y de su uso puede depender la vida de los pacientes; sin embargo, no está libre de riesgos, y aunque ha adquirido un gran desarrollo y seguridad, aún no es posible proporcionar un componente sanguíneo con riesgo cero².

Entre los efectos adversos más conocidos de la transfusión sanguínea se encuentran las reacciones febriles no hemolíticas, la hemólisis inmunitaria, la aloinmunización, la enfermedad del injerto contra el huésped, la inmunomodulación y las infecciones, como la producida por el virus de la inmunodeficiencia humana, el paludismo y las hepatitis $B$ y $C^{3,4}$.

La Norma Oficial Mexicana NOM 253-SSA-2012 «Para la disposición de sangre humana y sus componentes con fines terapéuticos" establece que el médico es el responsable de indicar una transfusión sanguínea, lo que vuelve imperativo que el profesional de la salud amplíe sus conocimientos sobre las indicaciones específicas, los riesgos, los beneficios y las alternativas. Una deficiente valoración que conduce a una transfusión innecesaria puede tener efectos sobre la salud y la seguridad del paciente ${ }^{5}$.

Se ha informado de que existe un aumento del número de transfusiones innecesarias, por lo que se han implementado diversas estrategias, como auditorías transfusionales, que pueden ser retrospectivas, concurrentes y prospectivas, y que tienen la finalidad de establecer un control de la transfusión. Diversas instituciones han publicado guías para mejorar la práctica transfusional y minimizar los riesgos de los efectos adversos. En México, al igual que en otros países, la transfusión de componentes sanguíneos se ha incrementado; por ello se han implantado las recomendaciones para la terapia transfusional de sangre y sus componentes con la finalidad de proporcionar a la población médica los conocimientos necesarios basados en evidencias que los conduzcan al uso correcto de los mismos ${ }^{6-11,13,14}$.

En México, el $4.8 \%$ de los casos de sida son secundarios a una transfusión de sangre, incluyendo pacientes hemofílicos. La hepatitis viral postransfusión es la complicación más frecuente y causa de un 80 a $90 \%$ de los casos de hepatitis C. Por lo tanto, el monitoreo de los efectos terapéuticos y adversos de la transfusión es un aspecto importante de la revisión sobre la utilización de la sangre?.

Cuando se limita el uso de componentes sanguíneos, se disminuyen los costos de operación del banco de sangre y se mejora el tiempo de trabajo de su personal; en hospitalización disminuye el consumo de material, y se evita el costo que genera el tratamiento de las complicaciones; los pacientes se favorecen porque reciben un tratamiento óptimo con un riesgo mínimo y a un menor costo; y finalmente, los médicos también se benefician porque prescriben un tratamiento apegado a los lineamientos establecidos, lo que disminuye su vulnerabilidad ante problemas médico-legales ${ }^{8-11}$.

El objetivo de este trabajo es conocer el apego de la transfusión a las recomendaciones vigentes en el Hospital de Alta Especialidad de Veracruz.

\section{Método}

Estudio de tipo cuantitativo y retrospectivo, el cual consistió en la revisión de las solicitudes de las transfusiones sanguíneas registradas en la base de datos, sobre los ingresos y egresos de sangre y sus componentes del banco de transfusión sanguínea del Hospital Regional de Alta Especialidad de Veracruz para el manejo de pacientes con deficiencia de los mismos. Se revisaron las solicitudes cursadas del primero de diciembre de 2013 al 30 de noviembre de 2014, con un tamaño de muestra calculada por el total de solicitudes captadas durante un año. Se incluyeron en el estudio todas aquellas solicitudes de transfusión que contaran con los siguientes datos: identificación del paciente, componente sanguíneo solicitado y número de unidades transfundidas, diagnóstico clínico y los siguientes estudios paraclínicos: hemoglobina, hematocrito, número de plaquetas, tiempo de protrombina y tiempo de tromboplastina parcial activado. Se analizó individualmente la indicación de la transfusión de cada una de las solicitudes registradas, tomando en cuenta los resultados de los análisis de laboratorio de los pacientes y considerándose la transfusión como adecuada o inadecuada de acuerdo con las recomendaciones de transfusión de la Asociación Mexicana de Medicina Transfusional (Tabla 1). El análisis de los datos se realizó mediante estadística descriptiva. 
Tabla 1. Indicaciones para la administración de componentes sanguíneos de acuerdo con dos guías transfusionales

\begin{tabular}{|c|c|}
\hline Componente sanguíneo & Recomendaciones de transfusión de la Asociación Mexicana de Medicina Transfusional \\
\hline Concentrado eritrocitario & $\begin{array}{l}\text { Anemia con signos y síntomas de hipoxia tisular no susceptible de tratarse por otros medios. } \\
\text { Hemoglobina preoperatoria }<8 \mathrm{~g} / \mathrm{dl} \text { en pacientes que serán sometidos a procedimiento quirúrgico } \\
\text { con alto riesgo de sangrado, cuando la anemia no tenga tratamiento específico y la intervención no } \\
\text { sea diferible. La dosis recomendada es de } 1-2 \text { unidades cada } 24 \text { horas, que se repetirán según la } \\
\text { condición clínica del paciente. }\end{array}$ \\
\hline Concentrado plaquetario & $\begin{array}{l}\text { La transfusión de concentrados plaquetarios en forma profiláctica debe considerarse en pacientes } \\
\text { estables bajo quimioterapia y mielosupresión con conteo de plaquetas }<10,000 / \mu \text { y en pacientes } \\
\text { que van a ser sometidos a procedimientos invasivos o cirugía con conteo de plaquetas }<50,000 / \mu l \\
\text { También debe considerarse en aquellos pacientes que presenten fiebre o infección con conteo de } \\
\text { plaquetas }<20,000 / \mu l \text {. La dosis recomendada en un adulto es de } 1 \text { concentrado plaquetario por } \\
\text { cada } 10 \mathrm{~kg} \text { de peso. }\end{array}$ \\
\hline Plasma fresco congelado & $\begin{array}{l}\text { Se recomienda transfundir plasma fresco congelado para la corrección de la deficiencia de } \\
\text { un factor de la coagulación (factores II, V, VII, IX, X, XI y XII) y reemplazo de anticoagulantes } \\
\text { naturales (antitrombina III, proteína C y proteína S). Para revertir en forma inmediata el efecto } \\
\text { de los anticoagulantes asociado a sangrado o la necesidad de un procedimiento invasivo o } \\
\text { quirúrgico de urgencia. Se puede condicionar su uso en transfusión masiva, enfermedad hepática, } \\
\text { ocasionalmente para proveer otros constituyentes del plasma (inhibidor de C-1 esterasa en el } \\
\text { edema angioneurótico hereditario). Se recomienda su uso en todo paciente con un índice de } \\
\text { coagulación > } 1.5 \text { que va a ser sometido a un procedimiento quirúrgico. }\end{array}$ \\
\hline Crioprecipitado & $\begin{array}{l}\text { Tratamiento de la hemofilia A cuando no se tenga la disponibilidad del concentrado de factor VIII. } \\
\text { Enfermedad de von Willebrand tipo } 1,2 \text { y } 3 \text { en ausencia de desmopresina (DDAVP), a excepción } \\
\text { del tipo } 2 \text { b. Hipofibrinogenemia. Disfibrinogenemia. Deficiencia del factor XIII. Uremia y sangrado, } \\
\text { o en ausencia de desmopresina (DDAVP). El reemplazo mediante crioprecipitados debe hacerse } \\
\text { tomando en consideración que cada bolsa contiene un promedio de } 100 \text { UI de factor VIII. }\end{array}$ \\
\hline
\end{tabular}

Se analizaron 3168 solicitudes de transfusión, de las cuales 2414 correspondieron a concentrado eritrocitario, 220 a concentrado plaquetario, 493 a plasma fresco congelado y 41 a crioprecipitado. Del total de las solicitudes de transfusión, 2131 (67.26\%) fueron adecuadas y 1037 (32.73\%) fueron inadecuadas. Según los componentes sanguíneos solicitados, 1550 (64.21\%) concentrados eritrocitarios fueron adecuados y 864 (35.79\%) fueron inadecuados; del total de concentrados plaquetarios, $86(39.09 \%)$ fueron adecuados y 134 (60.91\%) fueron inadecuados; los $493(100 \%)$ plasmas frescos congelados fueron adecuadamente indicados; y del total de crioprecipitados, $39(95.12 \%)$ fueron inadecuados y solo $2(4.88 \%)$ fueron adecuados (Tabla 2).

\section{Discusión}

Esto traduce la falta de conocimiento y apego a las guías tradicionales, y por ende se incrementa el riesgo que representa la transfusión de hemocomponentes, así como el dispendio de recursos. Todo ello obliga a que sea considerado un programa básico de medicina transfusional en las facultades de medicina, así como para los residentes en formación de todas las especialidades.
El análisis de resultados reportó que en un $67.26 \%$ de los casos la indicación transfusional fue adecuada y en un $32.73 \%$ fue inadecuada. Por componentes, el $60.91 \%$ de los concentrados plaquetarios y el $95.12 \%$ de los crioprecipitados fueron referidos inadecuadamente. La literatura muestra que hasta el $26 \%$ de los concentrados plaquetarios pueden ser inadecuados. En cuanto a las indicaciones de transfusión de concentrado plaquetario, una de las más relevantes es que los pacientes con conteos plaquetarios por debajo de 5000/mm3 y aquellos cuyos valores oscilen entre 5000 y 10,000/mm3 tienen alta probabilidad de hemorragia espontánea. En cuanto al uso de crioprecipitados, los pacientes pueden presentar sangrado microvascular difuso y fibrinógeno con valores $<100 \mathrm{mg} / \mathrm{dl}$. Los resultados obtenidos en el Hospital de Alta Especialidad de Veracruz en cuanto a las indicaciones de concentrado eritrocitario son mayores que los procedentes de la auditoría realizada por el Centro Nacional de Transfusiones, lo cual resulta bastante favorecedor, ya que es el concentrado sanguíneo más transfundido.

Debido a la falta de criterios clínicos para comenzar la terapia transfusional, una buena estrategia constituye la relacionada con un valor de hemoglobina de $6 \mathrm{mg} / \mathrm{dl}$; de no ser así, lo mejor es el monitoreo 
Gaceta Médica de México. 2017;153

Tabla 2. Proporción de los componentes sanguíneos administrados

\begin{tabular}{lccc}
\hline Componente sanguíneo & & \multicolumn{2}{c}{ Indicación transfusional } \\
\cline { 3 - 4 } & & Adecuada & Inadecuada \\
\hline Concentrado eritrocitario & 2414 & $1550(64.21 \%)$ & $864(35.79 \%)$ \\
Concentrado plaquetario & 220 & $86(39.09 \%)$ & $134(60.91 \%)$ \\
Plasma fresco congelado & 493 & $493(100 \%)$ & 0 \\
Crioprecipitado & 41 & $2(4.88 \%)$ & $39(95.12 \%)$ \\
Total & 3168 & $2131(67.26 \%)$ & $1037(32.73 \%)$ \\
\hline
\end{tabular}

de cada paciente para evitar la pérdida sanguínea hasta poder hacer la reposición de componentes sanguíneos, y que esta sea adecuada para evitar posibles efectos secundarios y pérdidas económicas, y que resulte lo más beneficiosa posible para el paciente. La realización de un constante monitoreo de cada solicitud de transfusión, la elaboración de guías para la terapia con componentes sanguíneos, las auditorías internas y la implementación de un programa básico de medicina transfusional reducirían los porcentajes de indicaciones transfusionales inadecuadas, y evitaría la aparición de reacciones adversas posteriores a la práctica clínica y de manera proporcional disminuirían los costos que esta conlleva.

\section{Conclusión}

En nuestro hospital, a raíz de los resultados encontrados se acordó de manera conjunta por nuestro Comité de Medicina Transfusional y el Departamento de Enseñanza llevar a cabo un programa de educación médica continua para el uso de sangre y sus derivados. Se continuará trabajando de la mano con el Comité de Hemovigilancia, el cual dará seguimiento a los resultados que se vayan obteniendo una vez concluido el primer curso de capacitación.
Exhortamos a todos los hospitales a que realicen auditorías periódicas para identificar irregularidades en la práctica básica y cotidiana de la medicina transfusional.

\section{Bibliografía}

1. Pliego Reyes C, Flores Alcantar G. Evolución de la transfusión sanguínea. Revista de la Facultad de Medicina de la UNAM. 2012;55:35-42.

2. Barba Evia J, Suárez Monterrosa E. Transfusión de paquete globular. Del beneficio clínico real a la inadecuada prescripción. Rev Latinoam Patol Clin Med Lab. 2015;62:46-54.

3. Flores Paredes W. Prescripción inadecuada de transfusión sanguínea en un hospital de referencia de Lima, Perú 2011. Rev Perú Med Exp Salud Pública. 2011;28:617-22.

4. Corbalá Fuentes $C$, Navarro Jaramillo F, Aguilar Solano A, et al. Análisis del uso clínico de la hemotransfusión. Rev Mex Patol Clin. 2003;50:104-8.

5. Tena Tamayo C, Sánchez González J. La transfusión sanguínea y los derechos del paciente. Revista CONAMED. 2015;10:20-6.

6. Juárez Rangel E, Vite Casanova M, Marin y López R, et al. Auditoría transfusional retrospectiva en el Centro Nacional de Transfusión Sanguínea. Revista de Investigación Clínica. 2004;56:38-42.

7. Barba Evia J. Transfusión de sangre y sus componentes: riesgos, beneficios e indicaciones. Rev Mex Patol Clin. 2004;51:97-108.

8. Cuevas Cisneros R, Acuña Mamani J, Grajeda Ancca P, et al. Criterios clínicos y laboratoriales usados para indicar paquete globular y plasma fresco congelado en el Hospital de Apoyo Departamental Cusco, 2006. SITUA. 2006;15:14-22

9. Malagón Martínez A, et al. Guía para el uso clínico de sangre. 3. ${ }^{\text {a }}$ ed. México: Secretaría de Salud; 2007.

10. Kaufman RM, Djulbegovic B, Gernsheimer T, et al. Platelet transfusion: a clinical practice guideline from the AABB. Ann Intern Med. 2015;162:205-13.

11. Carson JL, Grossman BJ, Kleinman S, et al. Red blood cell transfusion: a clinical practice guideline from the AABB. Ann Intern Med. 2012; 157:49-58.

12. Roback JD, Caldwell S, Carson J, et al. Evidence-based practice guidelines for plasma transfusion. Transfusion. 2010:50:1227-39.

13. Malagón A. Recomendaciones para la terapia transfusional de sangre y sus componentes. Gac Med Mex. 2002;138(Supl 1):S35-7.

14. Brecher M. Techincal manual. $14^{\text {th }}$ ed. American Association of Blood Banks; 2013. p. 451-80. 